\title{
Mondo de J.M.G. Le Clézio : Épreuves du monde orphelin
}

\author{
Majida Sayegh, \\ Université Libanaise, Facultés des Lettres et des Sciences Humaines \\ (Section 5), Département de Langue et Littérature Françaises, Lebanon
}

Doi:10.19044/esj.2020.v16n23p57～URL:http://dx.doi.org/10.19044/esj.2020.v16n23p57

\section{Résumé}

Selon une méthode exploratoire et analytique guidée par les données thématiques du récit, cet article étudie le cheminement de Mondo, fils de la nature, un enfant vagabond pas comme les autres. Tout en cherchant une famille adoptive, il apprend des leçons précieuses en compagnie des personnes possédant des caractères spécifiques qui enrichissent sa vision du monde. Parmi ces personnes, il y a des marginaux, et des autres qui sont déçues de ne pas pouvoir réaliser leurs rêves. L'alphabétisation se réalise dans une école en plein air qui s'accorde parfaitement avec les désirs profonds de Mondo de lire et d'écrire. Le pentagone qui illustre son nom, symbolise l'harmonie et l'équilibre naturels en suivant un temps circulaire dans le sens de l'aiguille d'une montre. La réussite finale de l'adoption non conventionnelle, qui se passe en pleine nature, auprès de Thi Chen, prouve la prédominance des accords d'âmes avant toute réglementation. Attrapé par les autorités, Mondo qui garde dans son cœur et pour toujours la valeur de cette adoption libre et sincère, préfère s'enfuir de cette civilisation prisonnière de ses propres principes.

Mots-clés : Enfance, identité, adoption, nature, apprentissage, liberté 


\title{
Mondo by J. M. Le Clezio: Ordeals of the Orphan World
}

\author{
Majida Sayegh, \\ Université Libanaise, Facultés des Lettres et des Sciences Humaines \\ (Section 5), Département de Langue et Littérature Françaises, Lebanon
}

\begin{abstract}
Using an exploratory and analytical methodology and following the themes of the story, this paper studies the journey of Mondo, nature's unique and vagabond son. While looking for an adoptive family, and accompanied by different characters, he learns valuable lessons and enriches his vision of the world. Some of such characters are marginalized by society, while others are disappointed from not having realized their dreams. Mondo's deep desires to learn to read and to write are perfectly fulfilled in an outdoor school. Illustrating his name with a pentagon creates a clockwise motion where time flows thus symbolizing a harmonious natural equilibrium. The final success in the non-conventional adoption, which happens in the wilderness with Thi Chen, proves the importance of the bonding of the souls before any kind of imposed laws and regulations. Caught by the authorities, and while still carrying in his heart the values of this free and sincere adoption, Mondo prefers to run away from a civilization shackled by its own principles.
\end{abstract}

Keywords: Childhood, identity, adoption, nature, learning, freedom

\section{Introduction}

L'œuvre "Mondo et autres histoires", est un groupement de nouvelles de Le Clézio (1978), "l'écrivain de silence » comme l'appelait Holzberg (1976) dans sa thèse, il se distingue par sa transparence et son symbolisme. Ce n'est pas fortuit que les enfants y jouent les premiers rôles qui reflètent une image de candeur couronnée d'espoir, raccourcissant la distance avec Adam qui « voudrai[t] bien vivre tout nu et tout noir (213)» (Le Clézio, 1963). Le Clézio ne fait-il pas un clin d'œil au père de l'humanité, selon la Bible ? Et que cette dimension de l'homme éclaire bien son identité partagée par les autres, et par suite, elle permet d'établir un fondement selon lequel «moi plus toi égale nous »?

En effet, depuis ses débuts littéraires, Le Clézio s'est distingué par sa vision philosophique qui insistait sur l'entité humaine avant tout : enfants, 
adolescents, adultes, vieillards, de races et de cultures diverses, sont les acteurs sur le théâtre de sa pensée universelle. Même au sein de la nature, les deux faces, les déserts et la verdure, sont mises à l'honneur comme deux milieux vitaux pour la vie, et Le Clézio y voit une complémentarité de l'essence de la beauté naturelle à condition que l'homme sache la spécificité de chacune d'elle.

La figure de l'enfant a une place primordiale dans l'imaginaire de Le Clézio : lors de son entretien avec Boncenne (1978), et à une question sur ses personnages «dramatiquement tendus vers l'avenir », il a répondu : « les enfants, dans Mondo, par exemple, sont arrêtés et assez heureux là où ils sont » [...] « J'ajouterais seulement que l'enfant, c'est moi ». En effet, le recours au personnage enfantin est la marque d'une préférence prononcée pour l'inné et le pure qui pourrait exprimer la transparence de la vie humaine à l'état naissant. Les réactions de l'enfance devant les difficultés de la vie sont illustrées dans leurs gestes et leurs langages qui sont souvent lacunaires et suggestifs loin de toute influence corruptrice de la civilisation. Dans "Mondo et autres histoires", selon (Manijashvili, 2014) « on rencontre des personnages qui s'expriment par des onomatopées : par des cris ... (le Mouiaaa! ou le Ya-ha-ho! des bergers) [...] Mondo échange quelques paroles avec ses amis, les marginaux ». Cet amour du gestuel et du silence est glorifié dans les nouvelles de Le Clézio car l'accent est mis sur le primitif archétypal qui interpelle chaque être humain dans son existence.

Que signifie le nom de Mondo ? Pourquoi Le Clézio l'a-t-il choisi pour cet enfant qui cherche une identité sans contraintes physiques et morales ? Sachant que dans l'esprit le clézien «l'identité n'est pas une donnée figée [...]. Elle procède d'un processus qui combine deux mouvements rhizomiques qui ne sont contradictoires qu'en apparence : déconstruire pour mieux reconstruire » comme l'énonce Diouf (2016). C'est pourquoi, le nom de Mondo est évocateur d'un processus à la recherche d'une identité unificatrice aimantée par le pouvoir de l'amour universel. En réalité, un nom est généralement choisi ou proposé par les autres, pour refléter un souhait, un espoir, c'est comme un anneau dans une longue chaine, ou comme disait Augé (1994 : 61) « le nom identifie l'individu en le rattachant à une filiation ». La filiation de Mondo est à reconstruire, à redéfinir : dans son ambiguïté même, ce nom est éloquent car il englobe tout enfant et tout avenir humain.

En effet, la géographie universelle dont surgit Mondo lui confère un aspect générique enrichissant : ce nom propre représente une entité complète de sens. Son aspect physique dénote un garçon sauvage mais «bon » instinctivement. Malgré son dénuement atroce, il n'est pas voleur ni agressif, il est souriant et rendait de petits services aux marchands pour survivre. D'ailleurs, ce n'est pas la nourriture qui est le centre de ses soucis : c'est l'adoption. A la recherche d'une personne qui l'accueillerait et qui pourrait 
répondre à ses besoins affectifs et intérieurs. La question « Est-ce que vous voulez m'adoptez ? (35) » réitérée plusieurs fois sonne comme un glas pour souligner l'acuité de ce problème qui l'accompagnait probablement dans tous ses vagabondages antérieurs.

La nouvelle liminaire annonce, haut et fort, une approche originale de la vie à travers le cheminement d'un enfant vagabond, Mondo, qui savait parler des choses simples «que les gens avaient oubliées, auxquelles ils avaient cessé de penser depuis des années (58)». Cet article vise à décrypter ces « choses simples » que Mondo exprimait ainsi que le message de Le Clézio à travers l'expérience de son protagoniste, volontairement énigmatique, caractérisée par le laconisme et le non- dit, une raison de plus pour se focaliser sur les images et les symboles effervescents qui émaillent cette nouvelle. En réalité, le récit de Mondo est une succession de scènes disparates, de tableaux, de figures humaines emblématiques, de répliques souvent lacunaires d'où la nécessité de démonter l'ensemble pour en extraire la signification profonde visée par son auteur. La méthode exploratoire et analytique utilisée à cet effet, consiste à scruter l'ensemble du texte afin d'en retirer les informations qui servent à répondre à la problématique du récit : la recherche du Mondo d'une identité perdue en exerçant une action quotidienne qui reflète les épreuves du monde orphelin. Pour cela, un grand effort est déployé pour rassembler, du texte étudié, des citations pertinentes sur lesquelles se basent les analyses préconisées. C'est une méthode scientifique qui permet une stratification adéquate de l'ensemble des informations visées afin de satisfaire les quatre sections de l'article (introduction et conclusion exclues).

Cette démarche vise à clarifier le cheminement progressif de Mondo surgissant de l'inconnu, débarquant dans une ville de la société moderne, vivant les aléas de l'existence et aspirant à se définir civilement et naturellement jusqu'à son départ définitif vers l'inconnu. L'accent sera mis sur les expériences capitales de Mondo : la vie fusionnelle avec la nature, les fréquentations significatives avec ses semblables, et surtout son alphabétisation hors du commun par le nettoyeur de la plage Marcel et l'adoption réussie de la part de Thi Chin dont la vieille demeure illuminée symbolise l'apothéose de ses rêves à la fois modestes et grandioses.

La ville qui accueille cet enfant malgré elle va témoigner de son expérience révélatrice de la vie en société. Si Mondo venait de nulle part estce pour rester dans cet état de déracinement identitaire et psychique ou seraitil à la recherche d'une destination qui satisferait ses aspirations ? Quel va-etvient pratique-t-il entre la nature, source d'idéal et d'harmonie, et la ville qui concrétise la réalité civile de la vie ? Dans quelle mesure les rencontres quotidiennes avec de divers personnages sont-elles un impact sur sa vie ?

En faisant l'hypothèse qu'il est à la recherche d'une identité plutôt rêvée qu'ordinaire, cet article vise à suivre Mondo dans ses pérégrinations 
quotidiennes afin de cerner les fondements qui l'accompagnent dans son cheminement. Réussira-t-il à atteindre son objectif? Autrement dit, l'adoption sera-t-elle couronnée de succès à la fin de son parcours? Mondo possédant la volonté et l'intelligence d'apprendre à lire et à écrire, mais, cherche-t-il une méthode d'apprentissage particulière qui rend la nature son véritable professeur ? Mais où trouvera-t-il la personne qui lui permettra de réaliser son souhait?

Pour y répondre, l'étude vise à mettre en lumière quatre sections qui ont balisé l'itinéraire de l'enfant-héros : la première section sera consacrée aux liens qu'il entretenait avec les éléments de la nature et leur rôle dans le façonnement de sa personnalité, et ceci comme un point de départ qui va déterminer la suite des événements. Tandis que l'expérience avec la société civile sera illustrée, dans la deuxième section, à travers les personnages fréquentés par Mondo. Ceux-là auront des rôles différents, non seulement, dans la vie quotidienne de ce garçon, mais aussi dans son cheminement affectif. L'un d'eux, le nettoyeur de la plage, se distingue par son rôle dans l'alphabétisation de notre héros, c'est pourquoi la troisième section lui sera consacrée, afin d'étudier la spécificité de cet apprentissage hors du commun. La quatrième et dernière section sera réservée à la phase ultime hautement symbolique dans la Maison de la Lumière d'Or et l'aboutissement de l'aventure de Mondo dans cette ville. Enfin cette recherche sera achevée par une conclusion.

\section{Mondo fils de la nature}

Dans la production littéraire le clézienne (Le chercheur d'or), (Le Clézio J.-M. G., 1985), Alexis partit pour découvrir les secrets de la nature à l'aide d'un jeune noir Denis, tandis que Mondo un "garçon d'une dizaine d'années, ... venait de très loin, de l'autre côté des montagnes, de l'autre côté de la mer (11) », en tant que découvreur et révélateur des secrets. Le choix des enfants comme personnages principaux de ses récits est lié certainement, en grande partie, à la personnalité de l'écrivain. En effet, peu de temps après la publication de son livre " Mondo et autres histoires", Le Clézio a déclaré lors d'un entretien (Boncenne, 1978) «Beaucoup de ce que j'écris maintenant, j'y avais pensé vers quinze /seize ans ». Ce qui révèle qu'un esprit d'enfance l'a guidé dans son cheminement littéraire : l'univers le clézien est comme le croit Holzberq (1981, p.148) « un univers enfantin, baigné de lumière et proche de la mer», où, « le calme est acquis sans effort, rien qu'avec le sourire d'un petit garçon ». Cette intimité entre l'enfance et la nature connote la franchise et l'innocence qui les caractérisent. L'apparition même de Mondo et la nonidentification de ses origines le rapprochent davantage de la nature-mère. Cherchait-il à s'en libérer ou au contraire à se dissoudre davantage dans ses 
éléments ? Il y était vraiment perdu ou cherchait-il à s'enfoncer davantage en invitant les autres à faire pareil ?

Mondo surgit de nulle part, on ne sait pas d'où il venait, et comment il avait arrivé par hasard dans cette ville. S'agit-il d'une expulsion forcée ou poursuivait-il un idéal en cherchant une adoption (12) ? Cherchait-il une notion particulière de la famille ? Certainement, cette idée trottait toujours dans sa tête et le faisait souffrir. Arrivant au gré des hasards dans cette ville, il en est devenu, et malgré sa situation de vagabond, un habitant, circulant dans ses rues, fréquentant ses marginaux et travaillant pour vivre. $\mathrm{Vu}$ ses expériences passées dans d'autres villes probablement, il était suffisamment souple dans son adaptation avec le nouveau milieu car « on s'est habitué à lui (11».

Mondo refusait de mendier et préférait travailler, mais en étant illettré, il avait besoin d'accomplir des tâches qui ne nécessitaient pas la connaissance de l'alphabet. C'est pourquoi il rendait des services aux maraichers qui en « ont toujours besoin pour décharger leurs cageots (12) ». En travaillant d'une camionnette à l'autre, cet enfant devient actif et trouvait un rôle utile à jouer dans la vie économique de tous les jours ce qui l'intégrait aux activités de la rue. A la fin du marché, il «se faufilait entre les étals (13)» pour ramasser quelques fruits : des pommes, des oranges et des dattes, et « les marchands aimaient bien Mondo, ils ne lui disaient jamais rien (13) ». Son but était simple : survivre sans se soucier du lendemain en ramassant l'argent par exemple. Au contraire, un morceau de pain lui suffisait largement car il trouvait toujours «quelques miettes pour donner à des amis mouettes (15)». Cet acte simple révèle un attachement aux oiseaux, message de liberté, et à la vie naturelle et harmonieuse qui ne peut pas être assurée que par le respect de la spécificité de la vie de chacune des créatures. Cet être qui avait « cette peau couleur de cuivre, et cette démarche légère, silencieuse, un peu de travers, comme les chiens (12) » finissait sa journée en quittant la société humaine et s'élançait vers son milieu naturel qui le protégeait : la mer était son ami le plus fidèle. La digue de pierre était sa maison en liberté, la plage inondée par le soleil promouvait l'harmonie avec la nature, en provoquant des lueurs des cristaux de sables et des galets lisses, les cachettes entre les rochers étaient son asile véritable. Mondo noue un lien intime avec la nature qui se montre comme un miroir de l'essence humaine. L'exil n'existe pas dans sa personnalité, car la liberté fortifie en lui le sentiment d'appartenance à la nature entière. Cela rejoint la pensée de Flores (1987) «L'homme est parfaitement inséré dans le monde et cela n'est pas un exil, mais un privilège ». C'est le cas de Mondo qui était parfaitement inséré dans le monde qui l'entourait : seuls les détenteurs des lois rigides de la société civile lui posaient un problème. Trouver des réponses aux interrogations de la société le torturait. 
En réalité, Mondo a constamment le souci de Ciapacan qui avait pour rôle de ramasser les enfants et les animaux perdus. Dormir la nuit dans des cachettes naturelles était pour lui une façon de se protéger contre ce danger et d'être en contact direct avec la tendresse de la nature qui ne le jugeait pas et ne lui posait pas de questions auxquelles il ne pouvait pas répondre. D’ailleurs, pour cet enfant, donner des informations sur sa propre identité était une mission impossible et une source de malaise.

C'est pourquoi, il fuyait toutes les interrogations, il parlait peu et adorait le silence comme un moyen de communication, un réconfort ultime pour une âme qui souffrait. Ainsi à travers Mondo, Le Clézio (1978:11) glorifie « Le chemin du silence ... J'essaie de marcher sur lui, de faire de lui ma route », et avec le silence, l'homme peut explorer beaucoup mieux soimême et selon Bruneau et Achaz (1973) « le sens commun se réfère souvent au silence : " Le silence est d'or", "Les grandes douleurs sont muettes" [...] les musiciens connaissent la valeur du silence ». C'est pourquoi, Mondo évitait les discussions et les questions qui appelaient des réponses et instaurait à leur place un dialogue selon son cœur avec les éléments naturels qui l'entouraient. Il ne ressentait aucunement l'exil car il était à sa place dans la nature cosmique qui lui offrait ses richesses et ses bienfaits. Il peuplait la nature de ses espérances et de ses rêves de voyage.

Pour Mondo qui était curieux, la plage n'était pas le vide : il inspectait tout ce qu'il voyait : « les coquillages incrustés dans le ciment (18) », le goémon vert, le bruit de vague, les étincelles de la mer, même parmi les blocs de ciment qui « avaient l'aire de gros animaux endormis, à moitié dans l'eau (18) », il préférait un et pour y s'asseoir, et « il lui parlait un peu, à voix basse, pour lui dire bonjour (18) ». Ce bloc lui suscitait la pitié car il semblait prisonnier, enterré à moitié par la terre, ne pouvant ni se déplacer, ni voyager. C'est pourquoi et pour le consoler, il « lui racontait même des histoires pour le distraire (18) ». L'échange s'avérait parfait car le bloc « aimait les histoires que lui racontait (18) ». La solitude de Mondo était féconde car elle lui permettait de dialoguer avec les composantes de la nature censées être muettes et insensibles. Cette attitude face aux pierres rappelle un vers du Naimy (2011(1943)), un écrivain et poète libanais qui décrivait la condition des hommes égarés dans la nature «Nous demandons au soleil de nous indiquer le chemin et nous plébiscitons même la pierre ». Ce dialogue indirecte et harmonieux avec la pierre connote d'un côté, le besoin de Mondo d'avoir un interlocuteur qui pourrait répondre à ses aspirations, et de l'autre, ce respect étrange de tous les éléments naturels animés par son amour de la vie. Il y trouvait même un abri chaleureux contre lequel il «s'allongeait en chien de fusil, la joue contre le ciment tiède, et il dormait un peu (18)». Position idéale pour le repos physique et psychique. Cette perception de la nature est soutenue 
par Chanvallon (2010) qui la voit comme « une source d'exploration des sens, parfois un refuge pour un repos social ».

Cette osmose avec le paysage maritime s'exprimait tous les jours : Mondo, ami de la plage, attendait chaque jour le lever du soleil en suivant ses différentes phases, son " auréole pâle (31) », son disque qui « apparaissait audessus de l'eau (31) », le moment où « la mer et la terre semblaient de la même couleur (31) ». En face de cette générosité lumineuse, il y avait les réverbères de la ville de « lumière pâle et fatiguée (31) » à l'image de la société civilisée dépourvue de la chaleur originelle du monde. Les sources naturelles lui semblaient généreuses et abondantes et surtout maternelles. Mondo vivait une sorte d'eurythmie avec le mouvement du soleil, en lui chantonnant le chant de son ami Cosaque devant les badauds, de l'autre jour « Ayaya, yaya, yayaya, yaya ... (31) ». Mondo pensait aux poissons qui « se réveillaient et bougeaient lentement sous leur ciel pareil à un miroir (32) », aux hippocampes qui «montaient le long des tiges d'algues pour mieux voir la lumière nouvelle (32) », même aux coquilles qui « entrouvraient leurs valves pour laisser entrer le jour (32)».

Ce dynamisme naturel des créatures qui vivaient en harmonie parfaite avec la nature l'habituaient à leur rythme et dans un mouvement presque mimétique, Mondo adoptait cette correspondance avec le cosmos. Ainsi les liens avec la mer devenaient profonds et rituels : chaque matin Mondo ôtait ses habits pour se réchauffer dans l'eau, se laver, se purifier. Il cherchait à voir au fond, une curiosité qui exprimait une passion pour le monde sousmarin en se plongeant et en ouvrant ses yeux dans l'eau « pour voir le fond (32) », et en entendant « une musique qu'on ne connait pas sur la terre (32)», qui est due au « crissement fragile de vagues qui déferlaient (32) ». L'élément aquatique prend ainsi toute sa valeur lustrale et vitale. Un va-et-vient s'instaurait entre l'eau et le soleil, source de chaleur et de lumière ouranienne : quand il sentait froid, il retournait à la plage pour chercher l'enveloppe du soleil. De cette manière, la plage devient l'abri véritable de Mondo et la plateforme à partir de laquelle les rêves et les espérances trouvent leurs essors.

De la plage, Mondo scrutait les régions environnantes et voulait « voir les arbres et les façades blanches des villas (32) » situées sur les collines, et éclairées par la lumière du soleil. Ce paysage composé de la terre ferme qui dominait la mer le subjuguait : la mer semblait plate sous la colline, et elle «brillait comme une plaque de fer-blanc (40)». Très attentif à tout ce qui se passait dans la nature et en sortant de sa cachette du soir, à la base de la digue, « Mondo voyait une grande fumée blanche qui s'étalait dans le ciel (39)». Y avait-il un incendie sur les collines, au-dessus de la ville, qui pourrait réduire en cendre les forêts de chêne-liège? Il était soucieux des éléments de la nature qui, dans sa générosité, contrastaient fortement avec les actions humaines qui les mettaient en danger. 
Cet attachement presque sensuel à la vie naturelle était perpétuellement animé par le désir de bouger, de se déplacer, en un mot de voyager au sein de cette nature pleine d'énigmes et de mystères. Le voyage était l'expression de sa curiosité innée qui se délectait des histoires de Giordan, le pêcheur, décrivant la vie fabuleuse là-bas, au-delà des frontières, dans un pays situé sur la Mer Rouge entre pêcheurs, palmiers, bateaux et requins. Sinon, pourquoi Mondo avait-il la passion des bateaux ? Oxyton était, à cet égard, l'emblème de ce désir : c'était un bateau « amarré au quai, au milieu des autres bateaux et il se dandinait sur l'eau (55) ». Mondo s'identifiait à ce bateau : il est prisonnier car attaché, il rêvait, peut-être, comme lui de large. Pour ce bateau il compose une chanson exprimant son désir de voyager et de pêcher en même temps :

\author{
Oxyton, Oxyton, Oxyton \\ On va s'en aller-er-er \\ On s'en va pêcher \\ On s'en va pêcher
}

Les sardines, les crevettes et les thons !

Du point de vue musical, nous sommes devant une rime qui est considérée «comme élément structurant de la chanson » selon la musicologue Catherine Rudent (Migliore, 2015). Certainement on serait complètement ému si on pouvait entendre Mondo en train de la chanter. Cette mélodie assez nostalgique reflète l'attachement intense de cet enfant au monde marin où « les requins sont toujours à la recherche des ordures qui tombent à l'eau, de quelque chose à chaparder. Mais ils ne sont pas méchants (21) ». N'est-ce pas un message pour vivre en harmonie sur la terre en respectant toutes les créatures vivantes ?

En effet, la passion de Mondo englobait tout le cosmos : son amour pour le ciel se manifestait le jour comme la nuit. Dans la lumière et dans l'obscurité totale. Le ciel pour lui est un monde peuplé de mystères aussi fascinant que la terre ferme ou le monde sous-marin. C'est un ensemble qui se complète. Mondo parlait du ciel comme s'il voulait résoudre ses mystères, il se manifeste comme un "réparateur de continuité" selon l'expression de Tritsmans (2005). Le fait de demander au peintre de dessiner le ciel est porteur d'une grande signification : rien ne peut remplacer l'espace vaste de l'élément aérien, source de lumière, de vie et de liberté. Ainsi, l'équilibre régnait dans son esprit à l'image de l'harmonie qui règne dans la nature au niveau de l'espace comme au niveau du temps. Cela répondait parfaitement aux aspirations profondes de Mondo ressenties instinctivement et dans toute leur transparence. 


\section{Les initiateurs de Mondo}

Amoureux de la nature qui le protège, Mondo n'est pas pour autant un sauvage qui vit à l'écart de la fréquentation des êtres humains qui participe à l'élaboration d'une qualité nouvelle des liens sociaux qui met l'accent sur la solidarité vue comme une valeur humaine par excellence. Peut-être, « il n'y a de solidarité qu'en vue de rendre l'autre et soi-même plus humains» comme disait Tanella (2011). Cette solidarité ne peut s'exercer que dans l'hétérogénéité et le pluralisme. En effet, il est courant chez Le Clézio de présenter des personnages « dont la première particularité est la différence » d'après Ben Aïssa (2013). C'est ce que nous allons découvrir dans cette section.

Hormis les deux hommes de la camionnette du Ciapacan, qui cherchaient des chiens et des enfants perdus, Mondo entretenait de bons liens avec les gens. Les hommes de Ciapacan représentaient le danger perpétuel qui le guettait pour le priver de sa liberté. C'est pourquoi il prenait garde et s'en cachait derrière les buissons quand c'était nécessaire. Il devait se méfier beaucoup d'eux et «c'est peut-être pour cela que Mondo trottait un peu de travers, comme les chiens (25) ».

Le marché avait permis à Mondo de nouer contact avec la société urbaine. Son but n'était pas de collecter de l'argent mais il voulait tout simplement gagner son pain à la sueur de son front, ce qui a favorisé en lui le sens de la dignité personnelle. Tout le monde l'aimait. Dans ses va-et-vient quotidiens dans les rues de la ville, il «avait trouvé beaucoup d'amis (15)». En passant devant chacun d'eux, il faisait « un clin d'œil ou un signe de la main (15) ».

L'arroseur public est l'un d'eux. Il offrait à Mondo un spectacle beau et amusant : son travail consistait à nettoyer la place des ordures tombées par terre en utilisant le jet d'eau et en provoquant un nuage des gouttes éparpillées sous la lumière du soleil et «on voyait des arcs-en-ciel légers au-dessus des voitures arrêtées (13) ». Mondo se comportait comme s'il attendait ce nuage qui, grâce à la lumière donnait naissance à l'arc-en-ciel avec ses fabuleuses couleurs. Tandis que les voitures arrêtées témoignaient de l'occupation des hommes et d'une vie pleine de contraintes. Ce nuage envoyait un message de liberté, d'espérance et de beauté et non un nuage vu comme "l'indécis, l'instabilité et le mouvement » selon Roussel-Gillet (2011). Grâce à ce phénomène naturel, Mondo et l'arroseur public échangeaient de signes de sympathie sans parler.

Parmi tous les commerçants, Mondo distinguait deux personnages féminins chez lesquelles il ressentait un échange maternel dont il avait certainement besoin. Rosa, « la grosse marchande de fruits (13)» lui donnait des pommes ou de bananes. Ida, la dame boulangère, lui assurait les morceaux de son pain quotidien. Cette dame représentait la source de tranquillité pour 
Mondo car elle jouait le rôle d'une mère nourricière en premier lieu. Elle lui permettait de travailler utilement en «portant du pain chez les commerçants du voisinage (15)».

La rencontre avec le pêcheur Giordan est marquée par la simplicité et la spontanéité : c'était sur la plage, ce pêcheur lui « avait fait un petit signe amical avec la main (19) ». L'homme avait préparé sa gaule en faisant soin de chacun de ses éléments : la ligne, le moulinet, l'hameçon, l'appât. Et dans un geste de gentillesse, «l'homme avait prêté sa gaule à Mondo (15) » pour lui apprendre la technique de la pêche sans rien lui demander. Mondo avait apprécié fortement l'initiative du pêcheur, et avec un grand plaisir, il «allait chercher pour lui des crabes pour appâter sa ligne (19)».

Le passage d'un cargo appelé «Erythria» engendrait toute une conversation rêveuse sur un pays lointain sur la Mer Rouge. Giordan allait plus loin dans sa réflexion en regardant «au loin vers l'horizon (21)», et il parlait « de petits bateaux de pêche avec des voiles en forme d'aile (21) », de partage du travail entre les hommes qui réparaient les filets et les enfants installés sur la côte en allumant « des feux sur la plage pour faire chauffer la poix qui sert à colmater les fissures des bateaux (21)». Giordan citait des villes de drôles de noms situées sur les côtes, ceci pendant la saison des pluies avec des tempêtes méchantes qui obligent les gens à rester chez eux. Il évoquait la personnalité d'un pêcheur qui vivait dans une île avec sa famille "dans une maison en feuilles de palmier, au bord de la plage (22) ». Sons fils aîné, fort intelligent, avait presque le même âge que Mondo, il accompagnait son père dans la pêche. Le ménage est heureux vivant en harmonie avec la nature, et nouant des liens d'amitié avec les autres qui vivaient dans les îles voisines.

Mondo était fasciné par le genre de vie de cette famille et rêvait en regardant l'avancement calme du bateau à l'heure du coucher du soleil (22). Pourquoi cette prolixité concernant la vie des pêcheurs sur l'autre côté de la mer? En effet, Giordan transmettait, indirectement, à cet enfant l'amour et le savoir-faire d'un métier naturel et originel : la pêche, ancien métier originel et lié au monde marin. Subjugué par ce monde décrit par le pêcheur, Mondo lui demanda : "Quand est-ce que vous irez là-bas ? (22)». Après avoir hésité dans sa réponse, et les signes de tristesse et de regret se manifestaient sur son visage, il répéta des mots discontinus « parce que...parce moi je suis un marin qui n'a pas de bateau (23)». Mondo fut marqué par cette réponse, et quand il commençait à rêver de voyager sur Oxyton, c'était avec Giordan qu'il souhaitait le réaliser car c'était un pêcheur privé de bateau.

Mondo avait fait aussi la connaissance de trois personnages : le Gitan et le Cosaque et leur vieil ami Dadi, "c'étaient les noms qu'on leur avait donnés (25) ». Le Gitan et le Cosaque représentaient l'émerveillement devant la musique et les tours magiques : amusement qui faisait sortir Mondo de la banalité de la rue en participant lui-même au spectacle en ramassant les 
mouchoirs tombés lors du spectacle de Gitan et en répondant favorablement à l'appel de Cosaque « Petit, aide nous à ramasser les pièces de monnaies (27) ». Mondo avait ainsi un rôle à jouer dans les distractions publiques. Mais sa préférence allait vers Dadi, un vieux vagabond qui lui inspirait la sagesse, le calme et la paix intérieure. En effet, ce personnage s'essayait au soleil sur un papier journal à côté d'une valise trouée et un couple de colombes appelés Pilou et Zoé.

Parmi les personnages dont la rencontre avait influencé Mondo était le champion des cerfs-volants. C'était un homme d'une cinquantaine d'années dont le cerf-volant «était comme un grand papillon jaune et noir aux ailes immenses (52) ». L'homme avait donné le fil et la bobine à Mondo en lui donnant aussi quelques conseils pour bien le commander. Ce champion lui avait promis de l'initier à ce sport lié au ciel qui le fascinait car il connotait l'élévation symbolique et la liberté des oiseaux. Mondo « pensait qu'il ferait le sien tout blanc, comme une mouette (54)».

Mondo avait aussi connu le rempailleur des chaises et son petit-fils Pipo. Il aimait cet homme car «c'était un homme vieux mais qui savait faire bouger ses doigts très vite pour entrelacer et nouer les brins de paille (57) ». Ces deux amis, le petit-fils et son grand père, évoquaient les métiers humbles liés au bonheur de travailler manuellement des matières naturelles qui renvoient à la simplicité de la vie. Ce métier n'apprend-t-il à l'homme la patience et le regard perçant ? Mondo aimait surtout Pipo parce qu'il « restait assis près de son grand-père, sans bouger (57) ». Il exprimait sa sympathie en lui offrant un cadeau formé de cailloux et autres objets de la mer que Pipo regardait longtemps en silence.

Ainsi, Mondo savait tirer parti de chaque rencontre avec les gens, même avec ceux qui étaient rencontrés fortuitement et rapidement : chaque expérience était une occasion de plus pour apprendre et accéder à un nouveau savoir qui l'enrichissait positivement.

Quant à la vieille personne qui ratissait la plage et qui ressemblait à un Indien, elle avait un rôle majeur dans la vie de Mondo. C'est pourquoi la section suivante lui est consacrée.

\section{L'alphabétisation fabuleuse}

Mondo qui « ne savait pas lire ni écrire (12)», était confronté tous les jours à ce manque et en souffrait. Pour un analphabète aussi curieux, la vie dans une ville où le support écrit est partout, est un tourment. Le fait d'aimer les illustrés et kit Carson et ignorer la lecture était une humiliation pour ce garçon qui était obligé d'avoir recours à des « retraités de poste qui fumaient leur cigarette en s'ennuyant (16) » pour se faire lire.

Deux situations avaient poussé Mondo à ne plus tarder dans cette quête du savoir : la première quand il demandait au facteur s'il y avait quelque chose 
pour lui, ce dernier avait sorti de sa besace « un petit journal imprimé » (59) et l'avais tendu au petit garçon gêné et perplexe. La deuxième quand il avait été grondé par Giordan étonné que Mondo n'arrivait pas à lire le nom du bateau «Erythrea » qui passait en lui disant « Tu n'as pas de bons yeux (20)». C'était dur de proférer ce genre de mots cruels à un garçon d'une dizaine d'années « tranquille, et de beaux yeux noirs (11) » et qui avait répondu « ce n'est pas cela, je ne sais pas lire (20) ». Cet incident désagréable était suffisant pour convaincre Mondo que Giordan n'était pas le maître recherché pour l'apprentissage de la lecture et de l'écriture bien que ce dernier s'était rattrapé en lui disant avant de partir en lui criant « Reviens me voir, je t'apprendrai à lire (23) ». Le mutisme de Mondo cachait un refus de cette proposition. Ainsi, son envie d'apprendre n'était que plus pressant, mais comment trouver la personne selon son cœur et qui aurait été prête à lui apprendre à lire et à écrire ?

Il ne cherchait pas n'importe quelle personne qui acceptait cette mission mais avant tout, il cherchait un type de personne qui pouvait répondre à certains critères, comme si Mondo attendait un signe de la nature pour choisir ce maître tant recherché. Jusqu'au moment où l'occasion s'était présentée d'elle-même : la rencontre attendue avec «ce vieil homme qui travaillait à égaliser la plage (privée) à l'aide d'un long râteau (59) ». Il ne connaissait pas son nom et même il ne le demandait pas. Le soleil avait laissé ses traces sur son visage, et «sa peau était tout usée et ridée comme celle d'un vieil éléphant (59) ». Mondo l'avait regardé attentivement, en s'interrogeant comment un homme à cet âge, exerçait-il un tel effort, sous un soleil ardent, en tirant les galets à l'aide de son râteau « de bas en haut de la plage (60) ». Le symbole de l'éléphant, évoqué par l'auteur, peut contribuer à la compréhension de ce personnage patient et travailleur. Pour Gnausch (2009) « l'éléphant est vénéré comme symbole de chance, de sagesse et d'une grande intelligence. Il est un nouveau symbole de paix pour le monde. Pour Poole (1996) « les éléphants sont connus pour leur intelligence, leurs liens familiaux étroits et leur complexité sociale, leur capacité inouïe de communiquer entre eux sur de longues distances ». En effet, ce vieil homme travaillait avec patience sans faire attention à personne et « la sueur coulait sur son visage (60) » et avec un mouchoir, il l'essuyait de temps en temps. L'homme qui « ressemblait un peu à un Indien (60) », était si calme et doux, son regard annonçait une lueur d'amusement. Il avait voulu se reposer contre le mur à côté de Mondo sans lui poser des questions sur son âge, son nom, son adresse, ses parents, parce qu'il « avait compris son interrogation (60) », il s'était contenté de lui dire « Salut ! (60) ». L'homme le regardait « comme s'il voyait à travers lui (60)».

Mondo qui « aimait bien ceux qui savent rester assis au soleil sans bouger et sans parler et qui ont des yeux un peu rêveurs (57) » l'observait avec patience, répliquait directement « je voudrais que vous m’appreniez à lire et à 
écrire s'il vous plaît (60) ». Pourquoi ce vieil homme avait-il été choisi ? Et pour quels critères ? Sans aucun doute Mondo avait ressenti un magnétisme naturel chez ce personnage qui partageait avec lui certaines caractéristiques. Et comme dans une perception extra-sensorielle qui s'instaure entre les deux et avec peu de mots, il lui demandait : «Tu ne vas pas à l'école ? (60)», Mondo répondit par le strict minimum avec deux mots « Non monsieur (60) $»$.

Et la première leçon commence instantanément dans une école pas comme les autres. La nature infinie, grande et ouverte avait remplacé la salle d'école formée de quatre murs. A l'aide de son canif, l'homme avait commencé à graver les signes des lettres sur des galets bien plats. En effet, les conditions de cet apprentissage donnent à réfléchir : l'enseignant et l'élève étaient assis sur le sable, le tableau était représenté par des galets de la plage, le stylo a été remplacé par un canif, et le creusement jouait le rôle de l'encre. Dans ce cadre naturel et sans aucune préparation préalable, le premier cours démarra en toute souplesse, comme si elle poussait spontanément de la nature elle-même.

A chaque lettre, le maître associait un rapport avec la nature. Par exemple la première lettre gravée $(\mathrm{A})$ ressemble à « une grande mouche avec ses ailes repliées en arrière (61) ». L'inspection de cette méthode d'apprentissage à lire et à écrire décèle l'importance de la nature qui invite les hommes à communiquer, avec bonheur, entre eux. Ainsi, chaque lettre devenait une histoire attrayante qui incite à la joie. À travers ces notions (61), on voit, la lune $(\mathrm{O}, \mathrm{C}, \mathrm{D})$, les montagnes $(\mathrm{M})$, les toits des maisons $(\mathrm{H})$, les vases $(\mathrm{U})$, l'éclair $(\mathrm{Z})$, les ventres $(\mathrm{B})$, les pattes $(\mathrm{P})$, les oiseaux $(\mathrm{V}, \mathrm{W})$, les serpents (S), les mouches (A), la dance (I, J), les arbres (L), les bateaux (T), le salut $(\mathrm{N})$, le secours $(\mathrm{Y})$ et les souvenirs $(\mathrm{X})$. C'était toute une philosophie de la vie harmonieuse avec la nature qui avait été évoquée par ce "vieil homme cassé !".

Les lettres ne sont plus des signes muets et neutres, mais ils deviennent étroitement associés à la nature et leurs rôles consistent à transmettre aux êtres humains le secret des éléments de la nature et la beauté du monde vivant. Autrement dit, les lettres deviennent l'instrument-même du grand maître " Nature". Ceci reflète la sagesse et la clairvoyance de cet éducateur original qui a su s'investir dans les potentiels cachés de l'apprenti en le sensibilisant à l'aide de la réalité naturelle qui l'entoure.

Cet ingénieux pédagogue applique directement sa méthode en choisissant quelques galets, en ajoutant un autre (61), en disant à Mondo, «Regarde. C'est ton nom écrit, là (61) ». Mondo impressionné de la concrétisation visuelle de son nom écrit devant lui, il dit «C'est beau ! (62)». Il y avait une association entre une montagne $(\mathrm{M})$, une lune $(\mathrm{O})$, « quelqu'un qui salue $(61 »(\mathrm{~N})$, un croissant de lune $(\mathrm{D})$ et encore une lune $(\mathrm{O})$ : MONDO. 
Mais la réaction spontanée de Mondo était « Pourquoi y a-t-il toutes ces lunes ? (62) ». La lune, présente trois fois, avait la plus grande part de son nom, et puis la montagne et le salut ne rappelle-t-il par le premier mot prononcé par le vieil homme quand il a vu Mondo ? C'est comme s'il y avait une association harmonieuse entre le ciel, symbolisé par la lune, et la terre, représentée par la montagne, tout cela dans une ambiance de paix que le mot « salut » connote. Ainsi, et par le biais des mêmes symboles, il apprit le nom de son maître Marcel, une façon vivante qui donne sens aux prénoms et les lie à l'esprit de la nature.

\section{La Maison de la Lumière d'Or}

Mondo souffrait de l'anonymat qui régnait dans les rues de la ville au milieu «des géants (38) ». Il avait l'impression d'être piétiné par la vie en marche ce qui avait attisé en lui le désir de s'en affranchir et d'accéder à la vision lumineuse de la colline. Malgré son amour pour la mer, ses yeux étaient souvent rivés vers les collines et sa « belle lumière qui brillait sur les façades des villas et rayonnait dans le ciel comme une auréole (41)».

En effet, cet endroit était à ses yeux un sommet de quelque chose, un but à atteindre, à comprendre car il était toujours préoccupé par le sort de ses forêts et la découverte de ses demeures jonchées là-haut comme des maîtres des lieux qui dominent la mer par leur regard altier.

Pour y accéder, le chemin était un escalier zigzagant, éprouvant et difficile à arpenter et n'avait rien d'harmonieux. Car parfois on avait l'impression de descendre au lieu de monter. Ceci connote les épreuves de Mondo et son chemin vers le bonheur. Il était fasciné par ce chemin tortueux et ardu qui «semblait conduire vers le ciel et la lumière (42)». Durand son ascension physique et symbolique, il n'était pas pressé : il ne courait pas, il prenait son temps, il vivait, il goutait la vie naturelle dans tous ses aspects. Le désir d'arriver ne l'empêchait pas de protéger l'essence de la vie «les brindilles et les graines (40) », comme s'il voulait mériter l'objet de sa quête : le sommet lumineux des collines. En arrivant à sa destination, il fut ébahi par « la lumière qui enveloppait la maison. C'était pour elle qu'il avait tout de suite donné ce nom à la maison, la Maison de la Lumière d'Or (43) ».

Le jardin négligé et presque abandonné de cette «belle maison ancienne (42) » ajoutait à son charme aux yeux de Mondo qui y pénétra à quatre pattes vu l'entrelacement des broussailles et des ronces. Scène qui rappelle l'entrée du Prince Charmant dans le jardin du palais de la Belle au Bois Dormant. Se sentant dans son milieu, il s'endormit en «chien de fusil (44) » entouré de « chaleur et de paix (44)». Cet instant marque un repos significatif et mérité : la fin d'une étape et un nouveau départ psychologique et presque spirituel. Le narrateur, à cet instant-là s'adresse directement à Mondo, comme pour souligner ces moments majestueux de transformations 
lumineuses et bénéfiques : «Quand tu dormais, Mondo, tu n’étais pas là. Tu étais parti ailleurs, loin de ton corps (44) ». En effet, un voyage s'était opéré dans la personnalité de Mondo dont le corps était endormi, mais sa pensée se promenait ailleurs, «parti dans la lumière chaude de la maison, dans l'odeur des feuilles du laurier, dans l'humidité qui sortait des miettes de la terre (44) ». Cette migration hors du commun souligne l'effet merveilleux de cet endroit enchanté par ses valeurs et ses symboles lumineux. Mondo endormi oublie la marginalisation dont il était la victime et accéda pour la première fois à cette place perdue symbolisée par l'intérêt porté à sa personne en devenant le centre des regards de toutes les créatures qui l'entouraient «Tout le monde te regardait, parce que tu avais les yeux fermés (44) ». Ce monde émerveillé qui l'entourait n'était que les créatures de la nature tant admirées par lui. C'était son tour d'être l'objet d'admiration et de glorification. Son amour était payé en retour : en effet, ces yeux fermés de Mondo allaient s'ouvrir à une réalité nouvelle qui marquait une étape cruciale dans son évolution.

En réalité, la rencontre avec Thi Chen fut hautement symbolique : le cadre, un jardin presque sauvage soulignait la nature dans laquelle allait évoluer cette connaissance avec la femme vietnamienne. C'était sa voix aiguë et calme qui le réveilla à la vie nouvelle. Avec sa petite taille comme une enfant, debout devant lui et en souriant elle lui demandait : «Qui es-tu ? Tu dormais ? (45) ». Son corps menu la rapprochait de lui et marquait la différence avec les passants des rues de la ville décrite comme des géants qui l'écrasaient de leur présence étouffante. En se faufilant dans la ville, Mondo se voyait si petit aussi par comparaison aux passagers bien habillés. L'encombrement des rues lui faisait peur surtout quand il les traversait. Cette image de la ville caractérise la vision le clézienne selon laquelle il y a une « surabondance d'impressions visuelles et auditives... un embouteillage est ainsi comparé à l'enfer, un carrefour devient un lieux d'assassinat, et partout l'homme est agressé par des caméras» (Boulos, 1999)

La douceur de cette femme se manifesta d'abord par ce réveil en douceur de Mondo qui essayait de s'excuser auprès d'elle, la dame avait répondu avec un sourire «Viens voir la maison aussi (45)». Cette attitude bienveillante soulignait les qualités de cette personne qui l'invitait à aller plus loin dans sa quête.

Après le thé, Thi essayait de poser une question à Mondo en l'aidant implicitement à la réponse : Tu n'es pas d'ici, n'est-ce pas ? (46) », pour cela, il répondait directement « Non, je ne suis pas d'ici (46)». Dès qu'elle ressentit qu'il n'avait pas de réponses, elle arrêta de le questionner, elle le comprit et s'harmonisa directement avec sa personnalité. En effet, la conversation souple et naturelle entre les deux fondait la base de leur compréhension mutuelle (47). L'essentiel était démarré et selon le cœur naturel innocent de Mondo, et les 
deux parlaient de la beauté de cette lumière du soleil qui illuminait perpétuellement son imagination (46).

La deuxième étape décisive fut quand Thi lui posa la question « $\mathrm{Tu}$ aimes ma maison ? (47) » et que Mondo répond «Oui madame (47) », sa réponse «Alors, c'est ta maison aussi, quand tu veux (47) » marque l'adoption véritable, tant recherchée par Mondo. Cette adoption passait par l'amour et le respect et non pas à travers le processus administratif habituel. En fait, Mondo avait posé la question d'adoption plusieurs fois, à des personnes qui lui plaisaient, mais il n'attendait pas la réponse. Peut-être «c'est comme s'il choisissait attentivement à qui il la pose. Lorsqu'il fut adopté par Thi Chin, il n'avait pas besoin de demander » comme disait Wilailak (2017). Ainsi, une nouvelle phase de paix s'annonça pour les deux : car l'adoption devait être ressentie des deux parties et non pas d'une seule, c'était un besoin mutuel. C'était le début d'un échange basé avant tout sur le non-dit.

Mondo dormait plusieurs nuits dans cette grande salle et parfois il dormait dans le jardin, comme pour témoigner de cette complémentarité entre la maison et le jardin, l'intérieur et l'extérieur, deux principes qui gèrent l'existence de l'être humain. Cette image prend la place qu'elle mérite dans la pensée de Le Clézio (1995:268) qui considère que "ce sont les plantes qui sauvent les hommes", et ce n'est pas étrange si Couplan (2005) l'a adoptée comme titre de son ouvrage. Ce monde intérieur était présidé par la dame qui lui montrait une grande affection, en lui racontant des histoires avant de s'endormir. Tandis que le monde extérieur était valorisé par les promenades durant lesquelles Thi lui tenait la main pour lui montrer des choses amusantes : des cailloux, des feuilles d'arbre, des graines rouges, des fleurs. Ils échangeaient des cadeaux, des scarabées noirs en échange des coquilles et des plumes de mouettes. Ceci reflétait la complémentarité des rôles et l'affection exprimée des deux côtés. La nature était mise en valeur, la maison n'avait pas emprisonné Mondo, au contraire elle l'attachait davantage à la pureté de la nature. Thi Chin ne lui avait rien imposé et elle le laissait libre, c'est pourquoi il était tellement heureux.

A ce stade, Mondo vivait autrement : il dormait bien, il mangeait des repas simples mais préparés avec amour. Un lien mère-enfant s'instaure entre les deux personnages qui commencèrent à goûter la paix et la sécurité. Le principe d'adoption retrouve son véritable sens : désir et besoin des deux partis dans une intention dénuée de tout intérêt matériel et gérée par l'affection et la sincérité mutuelle.

Ainsi rassuré et gardant sa liberté, Mondo se réveillait chaque matin pour se baigner à la mer et travailler comme d'habitude au marché où il glanait les fruits et les légumes pour le repas du soir avec Thi. Aidé par l'expérience rassurante de la Maison de la Lumière d'Or, Mondo était de plus en plus épanoui dans ces rapports avec ses amis qu'il cherchait continuellement même 
à travers les ondes qui «allaient vers eux, là où ils étaient, en se mêlant au bruit des vagues et à la lumière, et les gens les recevaient sans savoir d'où elles venaient (68) ». Ceci n'est-elle pas la conséquence logique d'un esprit ahurissant qui voit l'harmonie universelle dans l'interconnexion de tous les êtres ? La communication revêt ainsi un aspect presque sensuel où la matière s'enrichit du contenu psychique qui la meut. Le langage de cette communication dépasse toutes les barrières matérielles, un moyen d'atteindre l'essence de toute chose. C'est la notion du langage visée par Le Clézio, d'après Vogl (2005) «le langage ne représente qu'un des moyens à notre disposition pour sonder les mystères du monde».

Arrivant à la plage où il avait appris à lire et à écrire, Mondo grave les lettres de son nom sous la forme d'un pentagone, puis il les disposait sur le bord du mur, en espérant que son professeur Marcel les voyait «quand il viendrait, et qu'il sache qu'il était venu (69)».

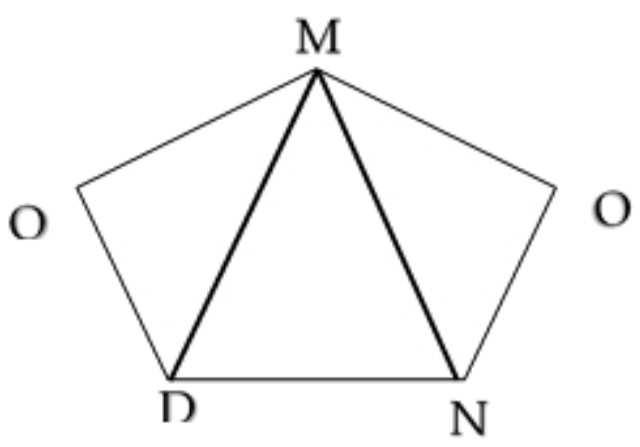

Décelant son identité perdue, les cinq lettres dans "MONDO" occupent les sommets d'un pentagone régulier et dans le sens de l'aiguille d'une montre. Dans ce pentagone, on retrouve le nombre d'or qui représente le plus grand secret de la nature. En effet, le triangle MDN est dit triangle d'or et le rapport $\mathrm{MD} / \mathrm{DN}$ est égale au nombre d'or, c'est une divine proportion dans l'histoire mathématique. Le nombre d'or s'observe aussi dans la nature, il est le principe naturel des lois de l'équilibre (Frost \& Prechter, 1978: 51-65). En écrivant son nom en suivant le sens de l'aiguille d'une montre, il voulait peut-être évoquer implicitement le rythme harmonieux de la vie avec le temps qui rythme tous les instants du cosmos. Ainsi, Mondo voulait laisser la preuve de son évolution concrétisée dans cette forme parfaite. Mais le bonheur ne fut pas de longue durée : cette harmonie originale entre amitié, affection et simplicité allait être cassée par les lois de la civilisation moderne. Mondo était traqué par le danger et la fin s'annonçait dramatique.

La fidélité de Mondo à son ami, le vieil homme Dadi, l'avait poussé à le chercher partout. Mais son chagrin était grand lorsqu'il avait appris par le Cosaque « qu'ils l'ont emmené cette nuit..., à l'hôpital (70), tandis que les 
colombes, « les oiseaux blancs (70) » disait le Cosaque, « peut-être qu'ils vont les manger... (70) ». Les quatre ailes des colombes n'existaient plus comme le rêve de Mondo qui se voyait emprisonné dans les rues de la ville. Ce fut un gros choc pour lui. Ce triste nouvel lui avait fait peur, en le rendant épuisé et inquiet. Il avait essayé d'aller dormir sur la plage, pour retrouver une partie de ses forces, mais il ne pouvait pas y aller.

A ce moment, Mondo «pensait à toutes ses bonnes cachettes au bord de la mer.... Ou bien dans le jardin de la Maison de la Lumière d'Or, (71) ». En réalité, Mondo pensait aux endroits où il goutait la paix et la liberté. Il tomba entre les mains du Ciapacan. Chaque centimètre de son corps était brûlant, et il imaginait des incendies qui réduisaient à néant toutes sortes d'arbres et de fleurs : ceci reflétait la fusion complète entre cet enfant et la nature. Le danger pour l'un ne saurait que mettre en danger l'entité de l'autre. Deux jours passés, et Thi Chin «était pâle et ses yeux étaient fatigués, parce qu'elle n'avait pas dormi (73) ». Elle avait su au commissariat de police que Mondo avait filé en mettant «le feu à son matelas (76) 》 à l'assistance publique. Mondo retourna à la case départ : pour garder sa liberté, il n'hésita pas à entraver les lois de la civilisation moderne. Il retourna à la cavale et à la bohème.

Mais les conséquences de cette disparition de Mondo étaient ressenties ailleurs, sur ses amis surtout : c'était la fin du Gitan, du Cosaque, et du Giordan qui avait cassé ses lignes. En sortant de l'hôpital, Dadi avait acheté un chat et il ne savait pas où sont partis ses deux colombes. « Le peintre de dimanche n'avait pas réussi à peindre le ciel, et il avait recommencé à dessiner des marines et des natures mortes (77) », et on avait volé le tricycle rouge au « petit garçon du jardin public (77) », et même la tristesse du bateau Oxyton était augmentée car il était toujours privé de sa liberté. Et son maître Marcel, « avait continué son travail en ratissant son morceau de plage sans partir pour les rives du Gange (77) ». En un mot, la part du rêve fut tuée dans toutes les âmes qui n'osaient plus s'envoler vers un monde ouranien libre et lumineux et se voyaient condamnées à un monde terrestre souffrant sous ses peines et sa finitude.

La présence de Mondo s’avéra une nécessité pour régénérer la vie comme il l'avait fait en désherbant le vieux jardin de la Maison de la Lumière d'Or, cet espace qui l'attendait et qui avait besoin de lui. C'était pourquoi, un jour, dans son jardin, Thi Chin avait trouvé des signes gravés sur des galets qui portent deux mots «Toujours Beaucoup (78)». N'est-ce pas une sorte d'onde envoyée par Mondo à Thi Chin pour affirmer qu'il l'aimait tellement et pour toujours ? Mais surtout un signe écrit que cet enfant révolté était déterminé à poursuivre son idéal et sa quête sans ne se soucier ni du temps ni de l'espace qui pourraient l'emprisonner dans leurs limites. 


\section{Conclusion}

Mondo voulant tenter sa chance dans cette ville, il s'était fait des amis avec qui il partageait tant de facteurs de marginalisation. Ceci l'ancrait dans la réalité dure de la rue en le poussant davantage à poursuivre sa quête d'adoption. Il avait réussi à se faire remarquer par des âmes généreuses qui l'avaient aidé à sortir progressivement du tunnel de l'ignorance en accédant simultanément à la paix de l'adoption et la reconnaissance symbolisée par Thi Chen et sa Maison de la Lumière d'Or.

L'épisode significatif de son apprentissage avec Marcel renvoie au problème éternel de la pédagogie et ses méthodes : Le Clézio insiste plus que jamais sur la nécessité d'éloigner tout abstrait en liant davantage l'apprentissage à la vie et à la réalité naturelle du monde.

C'est pourquoi, Mondo, fils de la nature, a accueilli très favorablement cette méthode souple et vivante en apprenant rapidement des techniques qui, dans une école traditionnelle, auraient besoin d'un temps incalculable afin d'être maîtrisées par l'apprenant. La preuve fut la gravure de son nom sous la forme d'un pentagone symbolisant 1'harmonie terrestre par excellence. Cela confirme la primauté du rôle de la nature en tant que professeur suprême de tout être humain et dans tous les domaines.

Par sa curiosité naturelle et son amour inné du savoir, Mondo a prouvé qu'un bohémien peut acquérir et partager tant de passions nécessaires à tous. Son expérience et sa fusion avec les éléments de la nature affirme la symbiose qui devait guider l'homme dans son appartenance à ce globe terrestre qui est l'identité de tout le genre humain et le facteur rassembleur qui unifie les cœurs au-delà des frontières. Ceci rejoint Özgüler (2016) « les déplacements multiples de l'écrivain à travers les trois grandes géographies culturelles (Europe, Amérique et Afrique) s'accompagnent aussi d'un voyage intérieur par le biais d'une écriture poétique faisant valoir l'autre, comme « le reflet de soi-même » et la diversité culturelle comme richesse et non comme fait de discrimination raciale ou de particularisme identitaire ».

A travers son personnage pur et innocent, Le Clézio désire recréer un mode plus harmonieux où l'espace et le temps suivent le même sens dans un symbolisme qui annule la discordance et favorise la convergence bénéfique pour le genre humain. Le sens de l'aiguille d'une montre serait le même sens de chaque atome dans l'univers : l'harmonie universelle se révèle non seulement matérielle et biologique, mais aussi psychique et intellectuelle. En donnant la forme parfaite au nom de son héros, l'auteur visait la perfection de la terre entière sans oublier aucun continent. $S$ 'inspirer de la nature semble le chemin le plus court pour sauvegarder en même temps l'être humain et la terre entière.

Il s'agit d'un message symbolique qui met en valeur la loi de l'équilibre qui règne dans la nature et qui incite les hommes à correspondre 
avec cette harmonie universelle en respectant le facteur espace-temps pour bâtir une civilisation moins matérielle et plus humaine qui vise au-delà des races, des ethnies, des croyances et surtout des langues. En effet, la langue ordinaire est remplacée chez Le Clézio par celle du silence car plus universelle et plus profonde. C'est dans ce sens que le mutisme de Mondo peut être compris.

Par son nom et son cheminement éthéré, Mondo est l'allégorie de la liberté humaine martyrisée dans une société qui a perdu le sens de la vie naturelle et authentique et s'est noyée dans le verbalisme et ne gardant de la réglementation que le principe de la contrainte contre laquelle Mondo a beau lutté. Cet enfant magique qui a su peupler le vide et animer l'inanimé, n'a pas réussi à s'imposer comme modèle de culture car la civilisation et ses lois l'ont emporté à la fin de la nouvelle : contraint de s'enfuir encore une fois devant les principes imposés par la société civile afin de préserver sa liberté. Ainsi l'hypothèse de l'adoption tant désirée par Mondo a été réussi selon les valeurs morales d'un nomade sans lequel, le spectacle de la vie quotidienne n'aurait jamais un sens complet car le monde homogène est ennuyeux et dévitalisé.

Ainsi, Le Clézio (1978 :257) invite le lecteur à entendre « le silence terrestre » qui fait réfléchir sur le pluralisme culturel guidé par un seul principe unique : respecter la vie globale dans la nature et ses créatures, et surtout dans tout ce qui est libre et spontané dans l'homme. Le Clézio lutte pour cette partie invisible et occultée par la modernité sans quoi l'être humain serait tronqué du principe vital qui est à l'origine du dynamisme de la vie humaine. Avec la disparition de Mondo, la part de rêve manque à la ville, et la créativité cesse en perdant l'âme en feu. Mondo, serait-il le bon barbare, dont la civilisation moderne avait tant besoin?

\section{References:}

1. Augé, M. (1994). le sens des autres. Actualité de l'anthropologie. Paris: Fayard.

2. Ben Aïssa, Y. B. (2013). Altérité et marginalité dans les ouvres de Jean-Marie Le Clézio et Amin Maalouf. Université Nantes Angers Le Mans et de l'Université de Manouba (L'unam), Thèse de Doctorat.

3. Boncenne, P. (1978). J.M.G. Le Clézio s'explique. Lire, 32.

4. Boulos, S. M. (1999). Chemins pour une approche poétique du monde. Le Roman selon J.M.G. Le Clézio. Museum Tusculanum Press, Kobenhavns Universitet.

5. Bruneau, T. J., \& Achaz, F. (1973). Le silence dans la communication. In: Communication et langages(20), 5-14.

6. Chanvallon, S. (2010). Anthropologie des relations de l'Homme à la Nature : la Nature vécue entre peur destructrice et communion intime. 
Thése de Doctorat, Université Rennes 2 ; Université Européenne de Bretagne. doi:https://tel.archives-ouvertes.fr/tel-00458244

7. Couplan, F. (2005). Ce Sont Les Plantes Qui Sauvent Les Hommes. Paris: Plon.

8. Diouf, A. (2016). Déconstruction-reconstruction identitaire et poétique de l'altérité dans Le procès-verbal de J-M G. Le Clézio. Voix plurielles, 13(2), 150-162.

9. FLORES, G. Á. (1987). J. M. G. Le Clézio ou la passion de la Terre. Revista de Estudios Franceses(3), 53-64.

10. Frost, A., \& Prechter, R. (1978). Elliott Wave Principle. New Classics Library.

11. Gnausch, R. M. (2009). L'éléphant: un nouveau Symbole de paix pour le monde. https://gnausch.net/efpwp/fr/qui-nous-sommes/pourquoi-lelephant/. Gnausch: Initiateur de «Elephants for Peace».

12. Holzberg, R. (1976). La Dialectique du silence dans l'œuvre de J.M.G. Le Clézio. Rutgers State Univ. of New Jersey-New Brunswick.

13. Holzberq, R. (1981). L'Eil du serpent : dialectique du silence dans l'œuvre de J.M.G Le Clézio. Sherbrooke: Naaman.

14. Le Clézio, J. (1978). L'Inconnu sur la terre. Paris: Gallimard.

15. Le Clézio, J. (1995). La Quarantaine. Paris, Gallimard (Fol. Paris: Folio.

16. Le Clézio, J.-M. G. (1963). Le procès-verbal. Paris: Gallimard.

17. Le Clézio, J.-M. G. (1978). Mondo et autres histoires. Paris: Gallimard.

18. Le Clézio, J.-M. G. (1985). Le chercheur d'or. Paris: Gallimard.

19. Le Clézio, J.-M. G. (2008). Peuple du ciel. Paris: Gillamard.

20. Manijashvili, I. (2014). le silence comme moyen de communication. Université d'Etat Iv. Djavakhishvili de Tbilissi, Géorgie, 149-156. Récupéré sur ojs.iliauni.edu.ge

21. Migliore, O. (2015). Chanson \& littérature : multiplier les approches . Acta fabula, " Musique ! On lit », 16(5). doi:URL : http://www.fabula.org/revue/document8224.php

22. Naimy, M. (2011(1943)). Le murmure des paupières (hams al-jofun), poème : La Route, ouvrage de poésie en arabe. Beyrouth: HACHETTE ANTOIN.

23. Özgüler, C. (2016). Les Enjeux De L'Interculturel Dans L'Oeuvre Romanesque De J.-M.G. Le Clézıo,. Université Hacettepe. Institut des Sciences Sociales. Département de Langue et Littérature Française. Thèse de Doctorat. Récupéré sur http://hdl.handle.net/11655/3606

24. Poole, J. (1996). l'étude des éléphants, l'éléphant d'Afrique, Nairobi,Kenya: publié par africain wildlife foundation. 
25. Roussel-Gillet, I. (2011). Textes brefs de Le Clézio : incertitudes et lueurs. Carnets: Revue électronique d'études françaises de l'APEF. Première Série - 2 Numéro Spécial 10-11, 67-80. Récupéré sur http://journals.openedition.org/carnets/5479

26. Tanella, B. (2011). solidarité et insécurité humaine : penser la solidarité depuis l'Afrique. Presses Universitaires de France " Diogène », 95-108.

27. Tritsmans, B. (2005). Figures du berger chez J.-M.G. Le Clézio et A. Dhôtel,. Nouvelles Etudes Francophones (NEF), 20(2), 57-68.

28. Vogl, M. B. (2005). Le Clézio en noir et blanc : La Photographie dans L'Africain. Nouvelles Études Francophones (NEF), 20(2), 79-86.

29. Wilailak, N. (2017). Mondo et le Ciapacan: J.M.G. Le Clézio et le rapport au monde. Journal of Humanities, 41(2), 30-39. 